\title{
A perfusion protocol for highly efficient transduction of intact pancreatic islets of Langerhans
}

\author{
A. R. Barbu • B. Bodin • M. Welsh • L. Jansson • \\ N. Welsh
}

Received: 9 February 2006 / Accepted: 8 June 2006 / Published online: 30 August 2006

(C) Springer-Verlag 2006

\begin{abstract}
Aims/hypothesis Successful gene transfer to pancreatic islets might be a powerful tool for dissecting the biological pathways involved in the functional impairment and destruction of beta cells in type 1 diabetes. In the long run, such an approach may also prove useful for promoting islet graft survival after transplantation in diabetic patients. However, efficient genetic modification of primary insulinproducing cells is limited by the specific compact structure of the pancreatic islet. We present here a whole-pancreas perfusion-based transduction procedure for genetic modification of intact pancreatic islets.

Materials and methods We used flow cytometry analysis and confocal microscopy to evaluate the efficiency of in vitro and perfusion-based transduction protocols that use adenoviral and lentiviral vectors expressing green fluorescent protein. Islet cell viability was assessed by fluorescence microscopy and beta cell function was determined via glucose-stimulated insulin secretion.

Results In intact rat and human pancreatic islets, adenoviral and lentiviral vectors mediated gene transfer to about $30 \%$ of cells, but they did not reach the inner cellular mass within the islet core. Using the whole-pancreas perfusion protocol, we demonstrate that at least in rodent models the
\end{abstract}

Electronic supplementary material Supplementary material is available for this article at http://dx.doi.org/10.1007/s00125-006$0390-5$ and is accessible for authorized users.

A. R. Barbu • B. Bodin · M. Welsh • L. Jansson • N. Welsh Department of Medical Cell Biology, Uppsala University, Uppsala, Sweden

\section{A. R. Barbu ( $\square)$}

Department of Medical Cell Biology, Biomedical Centre, P.O. Box 571, S-751 23 Uppsala, Sweden

e-mail: Andreea.Barbu@medcellbiol.uu.se centrally located insulin-producing cells can be transduced with high efficiency, while preserving the structural integrity of the islet. Moreover, islet cell viability and function are not impaired by this procedure.

Conclusions/interpretation These results support the view that perfusion-based transduction protocols may significantly improve the yield of successfully engineered primary insulin-producing cells for diabetes research.

Keywords Adenoviral vector Cell death $\cdot$ Islet .

Lentiviral vector

$\begin{array}{ll}\text { Abbreviations } \\ \text { ESM } & \text { Electronic supplementary material } \\ \text { GFP } & \text { green fluorescent protein } \\ \text { KRBH } & \text { Krebs-Ringer bicarbonate } \\ \text { PFU } & \text { plaque-forming unit }\end{array}$

\section{Introduction}

Much work is currently directed to finding highly efficient and non-toxic vectors for gene transfer in primary insulinproducing cells. Among these, viral vectors have emerged as first choice for engineering beta cells, mostly due to their capacity to mediate efficient gene transfer in non-dividing cells. Adenoviral vectors in particular offer the advantage of a high gene transfer efficiency as well as relatively longterm gene expression in primary beta cells [1]. However, one important limitation of the adenoviral-mediated gene transfer in beta cell research is the reduced ability to reach and transduce the inner cellular mass of the intact pancreatic islet. This is an important drawback for experimental designs that involve gene therapy approaches 
in which the final target for genetic modification is the insulin-producing cell, since, at least in rodent models, insulin-producing cells are centrally located in the core of the islet, surrounded by a mantle of non-beta cells at the periphery.

\section{Materials and methods}

Sprague-Dawley rats (Skanbur BK, Solna, Sweden) were anaesthetised with an i.p. injection of thiobutabarbital sodium (120 mg/kg body weight). A catheter (inner diameter $1.40 \mathrm{~mm}$ ) connected to a peristaltic pump was placed in the abdominal aorta so that the perfusion medium could flow freely into the pancreas. The gland was removed from the animals [2] and placed in a funnel at a constant temperature $\left(30^{\circ} \mathrm{C}\right)$. The gland was perfused for 45 to $60 \mathrm{~min}$ at a flow rate of $1 \mathrm{ml} / \mathrm{min}$ with a continuously gassed $\left(\begin{array}{lllll}95 \% & \mathrm{O}_{2}-5 \% & \mathrm{CO}_{2}\end{array}\right)$ Krebs-Ringer bicarbonate (KRBH) buffer, supplemented with $20 \mathrm{mg} / \mathrm{ml}$ BSA, $20 \mathrm{mg} / \mathrm{ml}$ dextran $\mathrm{T} 70$ and $0.3 \mathrm{mg} / \mathrm{ml}$ glucose, and containing $\sim 10^{9}$ plaque-forming units (PFUs) per pancreas of the green fluorescent protein (GFP)-expressing adenoviral vector, which corresponds to 20 to 50 PFUs/islet cell. In some of the experiments, the capillary endothelium was disrupted before administering the viral vector by preperfusion for $40 \mathrm{~s}$ with medium containing $0.1 \%$ Triton $\mathrm{X}-100$, followed by a 10-min wash with KRBH buffer only. Following transduction, islets were isolated and subsequently cultured for 2 to 7 days.

All other experimental procedures have been previously described $[3,4]$ or are given in the Electronic supplementary material (ESM).

\section{Results}

Adenoviral-mediated gene transfer to intact rat pancreatic islets

High-titre in vitro adenoviral-mediated transduction of intact islets reached efficiencies above 50\% (ESM Fig. 1). This approach, however, was paralleled by extensive cellular death [5] (ESM Fig. 1). Low-titre-mediated gene transfer into intact islets, on the other hand, resulted in 31\% GFPpositive islet cells and no major increase in cell death (Fig. 1 and ESM Fig. 1). This percentage was increased up to $48 \%$ if, prior to the transduction procedure, cell-to-cell contact of the intact islets was transiently disturbed by EGTA treatment. Confocal laser-scanning microscopy sections through adenovirus-transduced isolated rat islets showed that the cells transduced by the in vitro technique were located in the outermost cell layer, while the cells within the core of
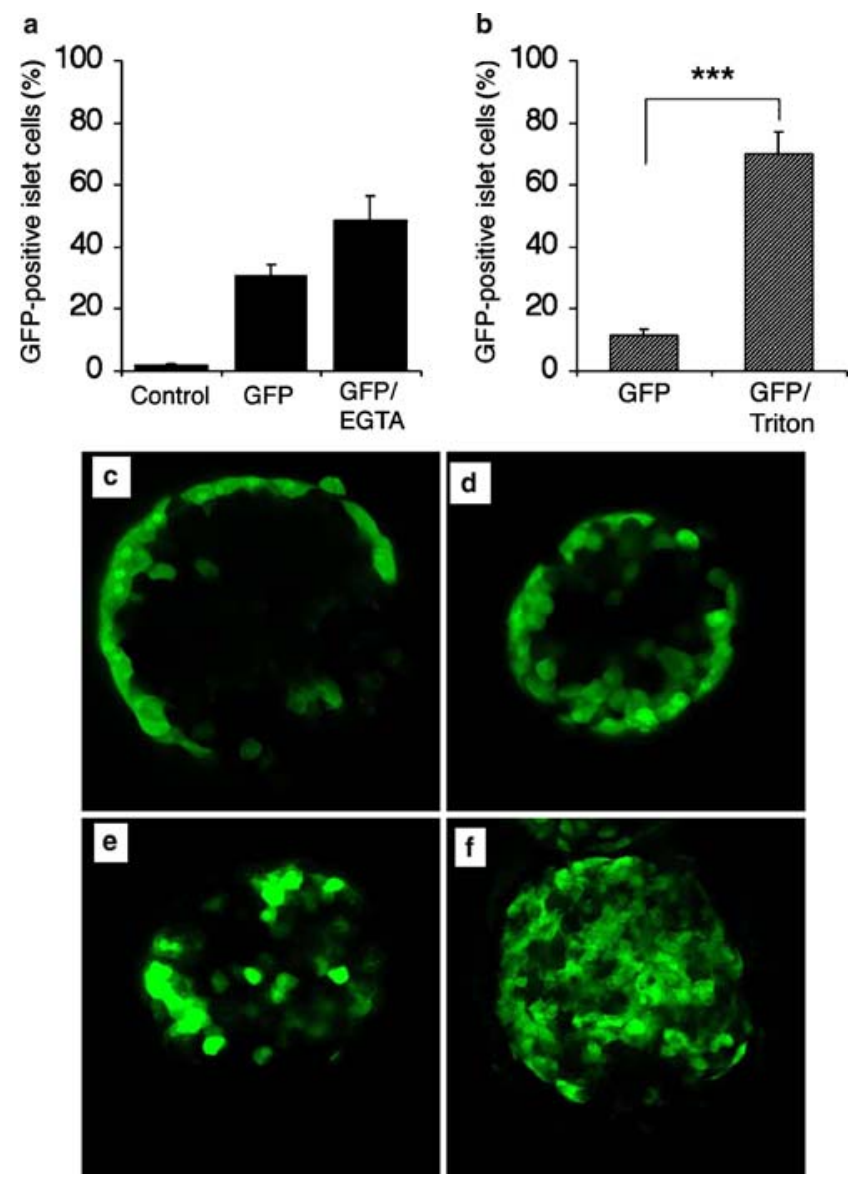

Fig. 1 GFP expression in rat islets following adenoviral-mediated transduction. a, b FACS analysis of dispersed islet cells expressing GFP. Rat islets were transduced in vitro (black bars) (a) or by the perfusion protocol (hatched bars) (b) with $50 \mathrm{PFUs} / \mathrm{cell}$ and then dispersed into a single-cell suspension. Results are means of six (in vitro) or four (perfusion) experiments \pm SEM. *** $p<0.001$ (GFP vs GFP/Triton) between the perfusion-mediated transduced groups. $\mathbf{c}-\mathbf{f}$ Optical sections at $50 \mu \mathrm{m}$ through an islet transduced in vitro (c), an islet transduced in vitro following incubation with 2-mmol/1 EGTA (d), an islet transduced using the perfusion protocol (e), and an islet transduced using the perfusion protocol after Triton X-100 treatment (f). Magnification $\times 40$

the islet were almost exclusively GFP-negative (Fig. 1). A similar GFP-positive cell distribution pattern was seen in the EGTA-pretreated rat islets (Fig. 1d).

EGTA-enhanced adenoviral- and lentiviral-mediated transduction of intact human islets in vitro

Having observed a modest increase in gene transfer efficiency using EGTA, we next investigated whether a similar approach affected transduction of intact human islets. Adenoviruses and lentiviruses were equally efficient in transducing intact human islets: $28 \%$ positive cells with the adenoviral vector and $30 \%$ with the lentiviral vector 
(Fig. 2a). However, analysis of optical sections revealed that lentiviral vectors were more efficient in reaching the cells located in the inner layers of the islets (Fig. 2d), while adenoviral vectors promoted gene transfer only in cells located at the periphery (Fig. 2b). Disruption of cell-to-cell contact by EGTA pretreatment resulted in a significantly improved transduction efficiency mediated by adenoviral

a

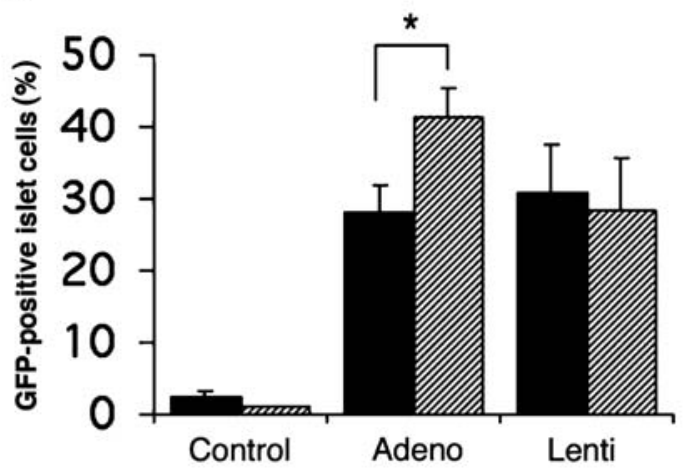

No EGTA

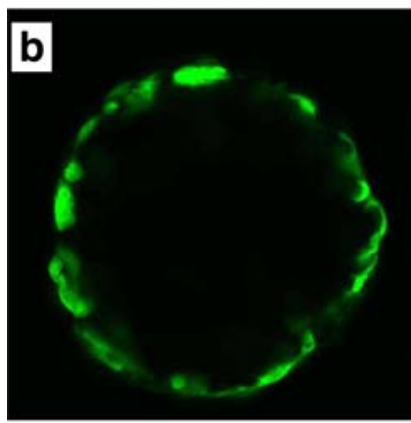

$2 \mathrm{mmol} / \mathrm{I}$ EGTA
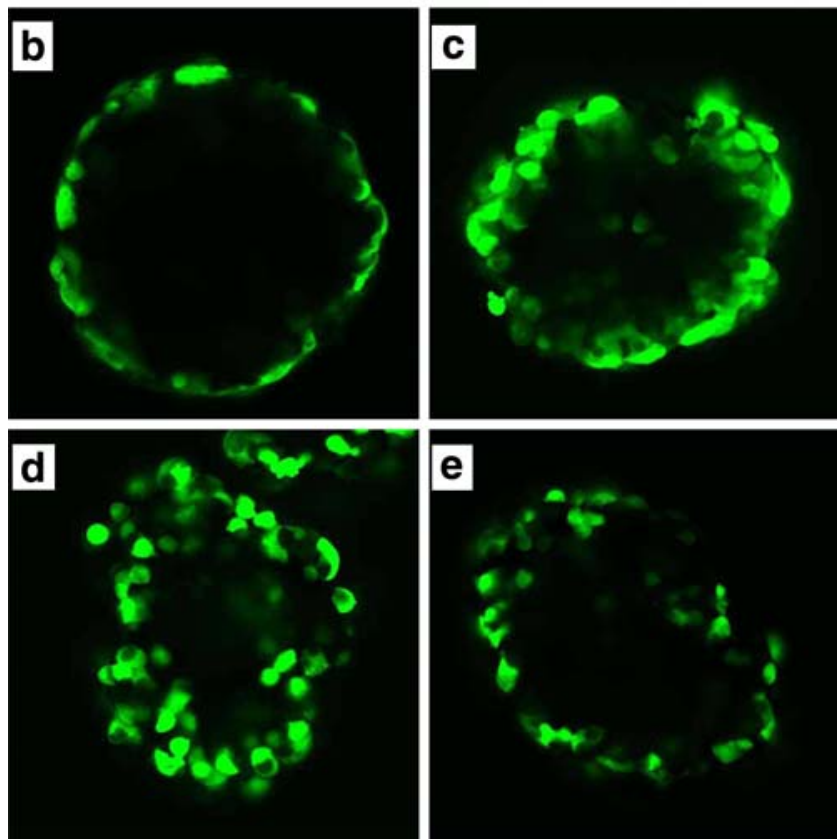

Fig. 2 GFP expression in human islets following adenoviral- and lentiviral-mediated delivery in vitro. a FACS analysis of dispersed human islet cells expressing GFP. Human islets were transduced in vitro with $100 \mathrm{PFUs} /$ cell of the adenoviral vector or with 200 transducing infectious units per cell of the lentiviral vector and then dispersed into a single-cell suspension. Results are means \pm SEM of four different experiments. ${ }^{*} p<0.05$ (no EGTA vs 2 mmol/1 EGTA) between the adenovirus-transduced groups. Black bars, no EGTA pretreatment; hatched bars, EGTA pretreatment. b-e Optical sections at $50 \mu \mathrm{m}$ through the adenoviral-vector-transduced islets (b), adenoviral-transduced islets following EGTA incubation (c), lentiviral-transduced islets (d), and lentiviral-transduced islets following EGTA incubation (e). Magnification $\times 40$ vectors but did not increase the outcome of lentiviralmediated transduction (Fig. 2a).

Perfusion-based adenoviral transduction of rat pancreas

As the majority of the islet cells cannot be reached by viral vectors, even though cell contacts have been disrupted by EGTA treatment, we next hypothesised that viral vectors delivered via the vascular system would more efficiently reach the islet core cells. When intact rat pancreases were perfused with $\mathrm{KRBH}$ buffer containing $\sim 10^{9}$ PFUs, only $11 \%$ of the islet cells were GFP-positive (Fig. 1a,b). However, as demonstrated by optical sectioning (Fig. 1e), the GFP-positive cells were dispersed throughout the islet rather than being confined to the periphery as in the in vitro approach (Fig. 1c,d). Disruption of the endothelium within the islet capillaries by Triton X-100 resulted in a significantly increased transduction efficiency, reaching more than $70 \%$ (Fig. 1a,b), accompanied by an even distribution of the positive cells both in the islet core and at the periphery (Fig. 1f). Additional insulin staining of islets isolated from the Triton X-100-perfused pancreases demonstrated that $65 \%$ of the GFP-positive cells were insulin-producing cells (ESM Fig. 2). The perfusion procedure had no influence on the number or size of the isolated islets. All islet preparations transduced by the perfusion-based technique and all islet sizes were characterised by a uniform distribution of GFP-positive cells. Moreover, the function and viability of perfusion-transduced islets were no different from the control islets (ESM Fig. 3). Nor was the viability and function of the in vitro virus-transduced human islets affected by the different treatments (ESM Fig. 4).

\section{Discussion}

Gene transfer to pancreatic islets is an attractive approach for elucidating basic mechanisms of beta cell function and destruction and could also provide a potential tool for improving islet graft survival following islet transplantation in type 1 diabetes [6]. Considering the compact structure of the pancreatic islet, a successful vector for gene delivery would have to be able to breach the outer surface of the islet and penetrate to the core. This is particularly important, since the rodent insulin-producing cell, the most obvious target for gene transfer in diabetes research, is preferentially located to the centre of the islet.

Here we show that in vitro transduction of intact rat and human islets using adenoviral vectors promoted GFP expression in only $30 \%$ of the cells. Moreover, by confocal sectioning of the intact islet, we also demonstrated that only the cells located in the outermost layer are successfully 
targeted. For some applications, such as immunoprotection of transplanted islets [6], low transfer efficiencies could suffice, provided that the transgene encodes a soluble protein. However, significantly higher transfection efficiencies are probably required for the study of specific mechanisms involved in the functional impairment/destruction of beta cells or when the induction of an intracellular protein (as anti-apoptotic proteins) is envisaged [1].

One option for increasing the transfection efficiency of intact islets is to dramatically increase the viral vector concentrations. However, both in dispersed islet cells [5] and in intact islets (present results), exposure to adenoviral vectors at high concentrations resulted in significant induction of cell death. In gene-therapy-based in vivo studies on type 1 diabetes, the toxicity exhibited by high concentrations of the viral vectors may be especially unfavourable because the vector itself could induce a virus-specific immune response or exacerbate an ongoing autoimmune attack.

Therefore, we evaluated alternative ways of exposing the inner islet cell mass to the gene transfer vectors. By transiently disturbing cell-to-cell contact and therefore increasing paracellular permeability, we observed an increase of the transfection efficiency. However, the distribution of GFP-positive cells within the islet was not dramatically modified, as the cells located in the core of the islet were still essentially GFP-negative. Instead, we next delivered adenoviral particles via the pancreatic arteries to the perfused pancreas ex vivo. Although the perfusion-based protocol resulted in rather low transduction efficiencies, the GFP-positive cells were distributed evenly in the core of the islet as well as at the periphery. We also observed that dilation of the pancreatic capillaries, promoted by glucose or adenosine, was not sufficient to allow efficient adenovirus passage from the blood vessels to the islet cells (results not shown). However, the number of islet cells expressing GFP was dramatically increased when the endogenous endothelium was disrupted by Triton X-100. This substance is known to selectively destroy the endothelium of the isolated heart, without affecting the smooth muscle layer [7]. Also in our system, the viability and function of islet cells exposed to Triton X-100 was unaffected.

Other recent publications have reported in vivo transduction of islet cells using i.v. or intraductal injections of viral particles in combination with clamping the pancreatic blood flow for 20 to $30 \mathrm{~min}$ [8,9]. These novel and interesting developments allow pancreatic transgene expression in vivo, but only at low levels, as the endothelium was not disrupted in these protocols. To the best of our knowledge, Triton X-100 and other endothelium-disrupting treatments are not tolerated in vivo, making highly efficient in vivo transduction not yet feasible.
The transduction protocol described here could provide a significantly improved yield of successfully ex vivo engineered beta cells, while keeping the specific pancreatic islet structure intact. This is of major importance, as preservation of the structural and functional integrity of genetically engineered pancreatic islets is a prerequisite for performing meaningful islet cell research. By ex vivo perfusion of intact human pancreases, followed by islet isolation and subsequent islet transplantation, a similar approach might be used for ex vivo gene transfer into human islet cells, as a possible tool for a successful application of gene therapy as a potential cure for type 1 diabetes.

Acknowledgements We thank G. Akusjärvi, Department of Medical Biochemistry and Microbiology, Uppsala University, Sweden for providing the adenoviral vector, and G. Mostoslavsky, Department of Genetics, Harvard Medical School and Molecular Medicine, Boston, MA, USA for providing the lentiviral vector. This work was supported by grants from the Swedish Research Council, the Swedish Diabetes Association, the Family Ernfors Fund, Barndiabetesfonden, Samariten Foundation, Lars Hiertas Minne Foundation, the European Foundation for the Study of Diabetes (Lilly) and the Novo Nordisk Fund.

Duality of interest The authors have stated that they have no dualities of interest.

\section{References}

1. Barbu AR, Akusjärvi G, Welsh N (2002) Adenoviral-induced islet cell toxicity is not counteracted by Bcl-2 overexpression. Mol Med 8:733-741

2. Jansson L (1986) Flow distribution between the endocrine and exocrine parts of the isolated rat pancreas during perfusion in vitro with different glucose concentrations. Acta Physiol Scand 126:533-538

3. He T, Zhou S, da Costa LT, Yu J, Kinzler KW, Vogelstein B (1998) A simplified system for generating recombinant adenoviruses. Proc Natl Acad Sci USA 95:2509-2514

4. Mostoslavsky G, Kotton DN, Fabian AJ, Gray JT, Lee JS, Mulligan RC (2005) Efficiency of transduction of highly purified murine hematopoietic stem cells by lentiviral and oncoretroviral vectors under conditions of minimal in vitro manipulation. Mol Ther 1:932-940

5. Barbu AR, Akusjarvi G, Welsh N (2005) Adenoviral-mediated transduction of human pancreatic islets: importance of adenoviral genome for cell viability and association with a deficient antiviral response. Endocrinology 146: 2406-2414

6. Lee M, Ko KS, Oh S, Kim SW (2003) Prevention of autoimmune insulitis by delivery of a chimeric plasmid encoding interleukin-4 and interleukin-10. J Control Release 88:333-342

7. Fort S, Lewis MJ (1993) A factor released from coronary vascular endothelium inhibits myocardial contractile performance. Am J Physiol 264:H830-H836

8. Ayuso E, Chillón M, Carcia F et al (2006) In vivo gene transfer to healthy and diabetic canine pancreas. Mol Ther $13: 747-755$

9. Wang Z, Zhu T, Rehman KK, Bertera S et al (2006) Widespread and stable pancreatic gene transfer by adeno-associated virus vectors via different routes. Diabetes 55:875-884 